\title{
Small perturbation analysis of diffracted holographic gratings
}

\author{
Ruey-Bing Hwang ${ }^{a}$, Ching-Chuan Wei ${ }^{b}$ \\ a Department of Communication Engineering, National Chiao Tung University, 1001 Ta Hsueh Rd., Hsinchu. Taiwan \\ ${ }^{b}$ Department of Electronic Engineering, Chien Kuo Institute of Technology, Chang Hua, Taiwan
}

Received 17 July 1995; revised 1 November 1995; accepted 8 December 1995

\begin{abstract}
Plane-wave scattering by a holographic grating is presented. The grating layer can be considered as a perturbation imposed on the uniform medium. Based on the perturbation analysis, this perturbation becomes the source of a diffraction wave and the boundary-value problem can be translated into the equivalent transmission-line equations with distributed current source; therefore, the Green function technique is utilized here to calculate the fields in the grating layer. The numerical examples are carefully compared and found to agree quite well with exact results obtained by rigorous treatment.
\end{abstract}

\section{Introduction}

A dielectric grating can be roughly classified into two kinds; one is the grating with spatial variation, and the other is the grating with medium variation. The holographic grating which we will treat in this paper belongs to the latter one. A grating with spatial-variation can be represented by a Fourier series. On the other hand, a grating with medium variation can be regarded as a first-order approximation of a spatial-variation grating. Our interest here is to exploit the basic feature for the method of small perturbation.

The analysis of a dielectric grating has been presented by many authors. To mention a few, the rigorous formulation of the diffraction grating was presented in the past $[1-3]$ and the exact analysis of a grating coupler has been presented in Refs. [4,5]. In addition, the approximate method of boundary grating diffraction coefficients was used to examine diffraction gratings [6].
For the research of perturbation analysis in gratings, there has been a continuing study for developing a better approach. For example, an improved perturbation analysis of dielectric gratings is given by Handa et al. [7]. Also, Zhang et al. [8] reported a design of broadband grating couplers by using perturbation analysis. In this paper, the characteristics of a diffraction grating instead of a grating coupler will be investigated.

The scope of this paper is as follows. Section 2 describes first the physical model and all the relevant parameters for the ensuing analysis. The mathematical procedure is outlined in Section 3 and the overall diffraction phenomenon can be reduced to the problem of an equivalent transmission-line equation with distributed current source. The numerical examples and conclusions are given in Section 4, with a particular emphasis placed on the non-Bragg-regime operation, and the diffraction efficiency of the 1st order is compared with that given by an exact formulation $[2,3]$. 


\section{Statement of the problem}

The perturbation method is presented for the analysis of a plane-wave scattering by a holographic grating. The grating is periodic in the $x$ direction with dielectric constant $\epsilon_{2}(x)$ and uniform in the $y$ direction. The upper and lower half space will be referred as the substrate and air region with the dielectric constants $\epsilon_{3}$ and $\epsilon_{1}$, respectively, as shown in Fig. 1. The thickness of the grating is designated as $t_{g}$ and the dielectric constant of the grating layer is given by:

$\epsilon_{2}(x)=\overline{\epsilon_{2}}+\epsilon_{p}(x)$,

where the periodic function $\epsilon_{p}(x)$ is regarded as the perturbation term, and can be written as:

$\epsilon_{p}(x)=2 \delta \overline{\epsilon_{2}} \cos (2 \pi x / d)$,

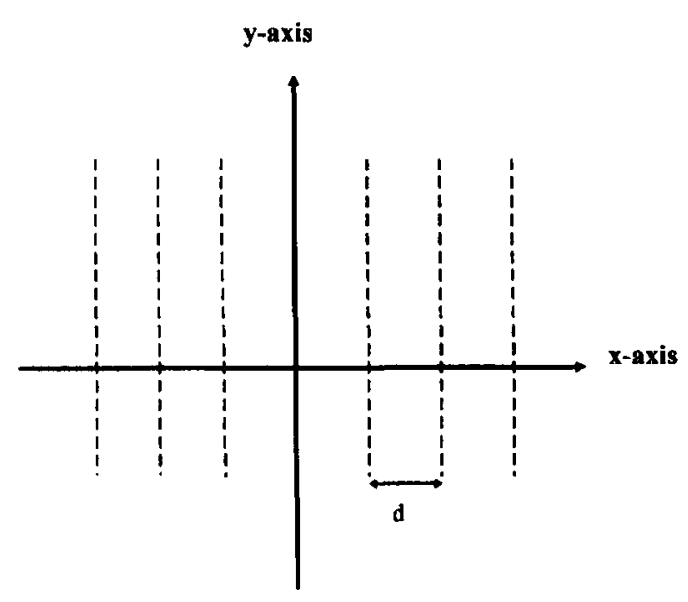

(a) Top view

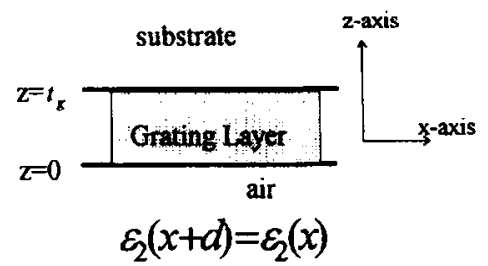

(b) Front view where $\delta, \overline{\epsilon_{2}}$ and $d$ are, respectively, the modulation index, the average dielectric constant, and the period of the grating. However, a dielectric grating with spatial variation can be represented by a Fourier series and the model of holographic grating with modulation medium can be regarded as its first-order approximation.

\section{Method of analysis}

In the previous section, the relevant parameters of the holographic grating have been defined clearly. In this section, the plane wave is incident from the air into the grating region. We assume that the electric field of the incident plane-wave is in the $y$ direction. The electric and magnetic fields in the grating layer can be written as the decomposition of unperturbed and perturbed terms $[7,8]$, corresponding to $\epsilon_{2}(x)$, and are given by:

$E_{y}(x, z)=E_{y}^{u}(x, z)+E_{y}^{p}(x, z)$,

$H_{x}(x, z)=H_{x}^{u}(x, z)+H_{x}^{p}(x, z)$,

where $\left(E_{y}^{u}(x, z), H_{x}^{u}(x, z)\right)$ denote the unperturbed fields, and $\left(E_{y}^{p}(x, z), H_{x}^{p}(x, z)\right)$ refer to the perturbed fields caused by the periodic perturbation $\epsilon_{p}(x)$. The unperturbed field quantities can be easily obtained by solving the boundary-value problem of multi-uniform layers. Therefore, the field quantities are assumed known and given by:

$E_{u}(x, z)=V_{0}(z) \exp \left(-j k_{x .0} x\right)$

$H_{u}(x, z)=I_{0}(z) \exp \left(-j k_{x, 0} x\right)$,

where $\left(V_{0}(z), I_{0}(z)\right)$ represent the unperturbed amplitudes in the $z$-direction and $k_{x 0}\left(=k \sin \theta_{i}\right)$ is characterized by the propagation constant $k$ of the incident wave and incident angle $\theta_{i}$. Because of periodicity, the perturbed fields in the grating layer can be written as:

$$
\begin{aligned}
& E_{p}(x, z)=\sum_{n=-\infty}^{\infty} V_{n}(z) \exp \left(-j k_{x, n} x\right), \\
& H_{p}(x, z)=\sum_{n=-\infty}^{\infty} I_{n}(z) \exp \left(-j k_{x, n} x\right),
\end{aligned}
$$

Fig. 1. Structure configuration of a grating. 


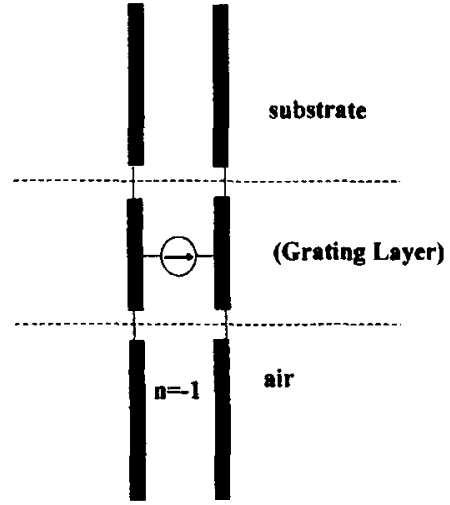

Fig. 2. Equivalent transmission line with distributed current source.

where $V_{n}(z)$ and $l_{n}(z)$ for $n= \pm 1, \pm 2, \ldots$ denote (voltage and current) amplitudes in the $z$-direction, and $k_{x, n}$ is the propagation constant in the $x$-direction, which is given by:

$k_{x, n}=k_{x, 0}+2 \pi n / d$.

The next step is to introduce Eqs. (5), (6), (7) and (8) into (3) and (4). Thus, we can obtain the equivalent transmission-line equations with distributed current source, as shown in Fig. 2, and described by:

$-\partial V_{n}(z) / \partial z=j k_{z, n}^{(i)} Z_{n}^{(i)} I_{n}(z)$,

$-\partial I_{n}(z) / \partial z=j k_{z, n}^{(i)} Y_{n}^{(i)} V_{n}(z)+J_{n}^{(i)}$,

where $Z_{n}^{(i)}=\omega \mu_{0} / k_{z, n}^{(i)}, \quad Y_{n}^{(i)}=1 / Z_{n}^{(i)}$ and $k_{z, n}^{(i)}=$ $\left(k_{0}^{2} \epsilon_{(i)}-k_{x, n}^{2}\right)^{1 / 2}$ for $i=1,2$ and 3 , and the distributed current source denoted by $J_{n}^{(i)}$ is written as:

$J_{n}^{(i)}=0 \quad$ for $i=1,3$,

$J_{n}^{(i)}=j \omega \epsilon_{0} \overline{\epsilon_{2}} \delta V_{0}(z)$ for $i=2$.

The corresponding boundary conditions are given by:

$$
\begin{aligned}
& -j \omega \mu_{0} \frac{\left.V_{n}(z)\right|_{z=0}}{\partial V_{n}(z) /\left.\partial z\right|_{z=0}}=Z_{n}^{(1)}, \\
& -j \omega \mu_{0} \frac{\left.V_{n}(z)\right|_{z=t_{g}}}{\partial V_{n}(z) /\left.\partial z\right|_{z=t_{g}}}=Z_{n}^{(3)} .
\end{aligned}
$$

After a substitution process, Eqs. (10) and (11) can be expressed as a second-order differential equation, as shown in the following:

$$
\left(\mathrm{d}^{2} / \mathrm{d} z^{2}+\left(k_{z, n}^{(2)}\right)^{2}\right) V_{n}(z)=j \omega \mu_{0} J_{n}^{(2)}(z),
$$

with boundary condition as the same as (13a), (13b). Therefore, the voltage wave can be obtained by the following formula:

$V_{n}(z)=j \omega \mu_{0} \int_{0}^{t_{g}} J_{n}\left(z^{\prime}\right) G_{n}\left(z, z^{\prime}\right) \mathrm{d} z^{\prime}$,

where $G_{n}\left(z, z^{\prime}\right)$ is the Green function corresponding to (14) with delta current source and the mathematical derivation is listed in the appendix. Fortunately, the analytical integral can be obtained and save much CPU-time for numerical computation. Eventually, the diffraction power is proportional to $V_{n}(z) I_{n}^{*}(z)$, which can be easily calculated.

\section{Numerical results and conclusions}

In this paper, the average dielectric constant $\overline{\epsilon_{2}}$, $\epsilon_{3}$ and $\epsilon_{1}$ are assumed to be $1.44,1$ and 1 , respectively. The perturbation analysis is compared with the exact solution in the figures. In Fig. 3, the grating thickness is varied and the diffraction efficiency between exact and perturbation analysis almost matches. Also, we can observe a fast-variation curve resulting from multiple-reflections. In the next

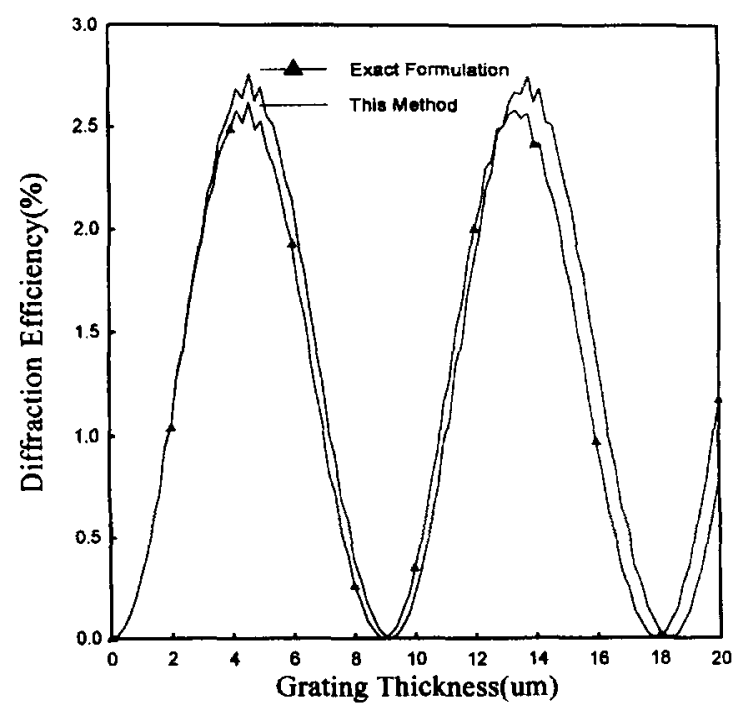

Fig. 3. Comparison of perturbation and exact methods against various grating thicknesses. The modulation index $(2 \delta)$ and the ratio of the grating period versus wavelength are 0.01 and 2 , respectively. 


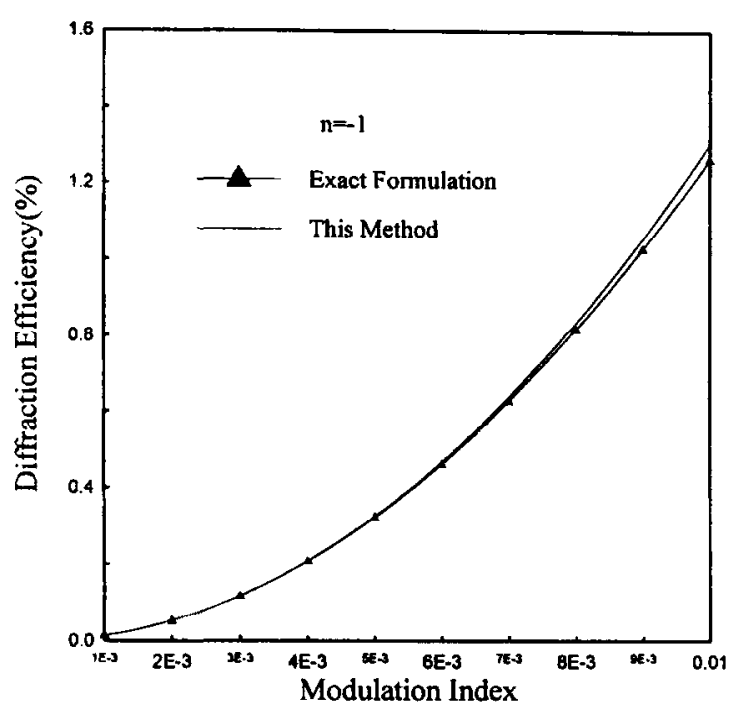

Fig. 4. The diffraction efficiency of a normal incident wave is calculated for various modulation indexes. The grating thickness and the ratio of grating period versus wavelength are $5 \mu \mathrm{m}$ and 2 , respectively.

example, the modulation index $(2 \delta)$ varies from 0.001 to 0.01 and the result is shown in Fig. 4, where it reveals less deviation between exact and perturba-

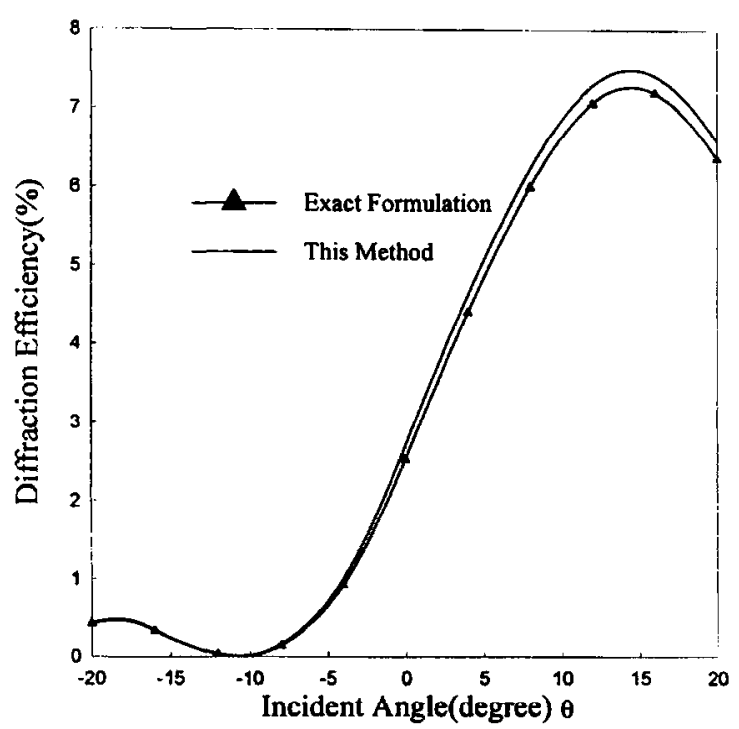

Fig. 5. The diffraction efficiency is plotted against various incident angles. The grating thickness, modulation index and the ratio of grating period versus wavelength are $5 \mu \mathrm{m}, 0.001$, and 2 , respectively.

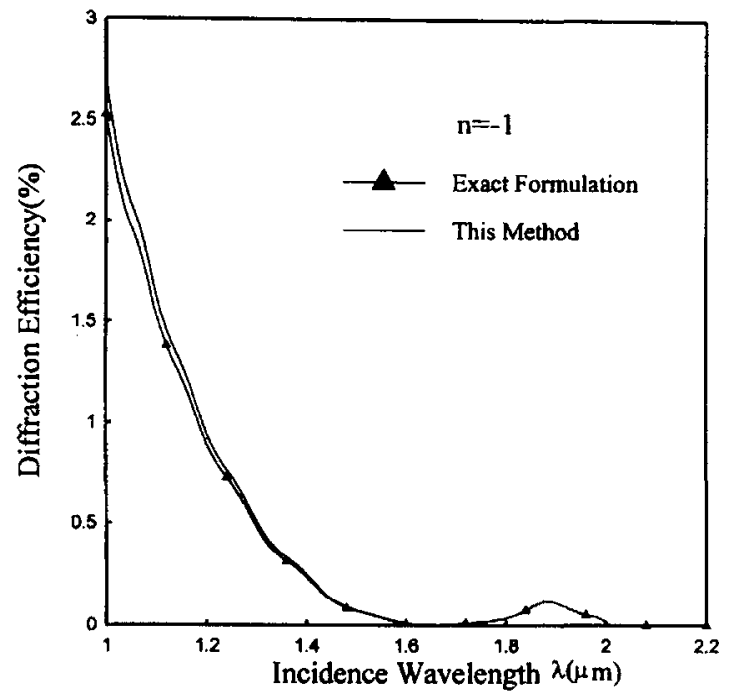

Fig. 6. The diffraction efficiency versus wavelength: modulation index $(2 \delta)=0.01$, grating thickness $t_{g}=5 \mu \mathrm{m}$.

tion solution. In Fig. 5, the incident angle is variable and the maximum diffraction efficiency occurs at 14.5 degrees, which is the first-order Bragg condition. In Fig. 6, the curve shows that the diffraction power vanishes when the wavelength is larger than 2 , and this is reasonable because the first-order diffracted wave is below cutoff as $\lambda / d \geq 1$.

Using the concept of first-order perturbation theory, the diffraction efficiency of a holographic grating is analyzed through an equivalent transmissionline network and Green function technique. Compared with exact solutions, the results of the approximation method have excellent agreement. To sum up, this analysis is easy and provides us with a new view point to find the physical insight about the diffraction of a holographic grating.

\section{Acknowledgements}

The authors express their sincere gratitude to the reviewers and editor, for their encouragement, critical reading of the manuscript, and many suggestions for improvement. 


\section{Appendix A. Green's function technique}

The Green function technique is applied to solve the differential equation (14) with boundary conditions (13a) and (13b), i.e., the problem is transformed into solving the following differential equation,

$\left(\mathrm{d}^{2} / \mathrm{d} z^{2}+\left(k_{z, n}^{(2)}\right)^{2}\right) G_{n}\left(z, z^{\prime}\right)=\delta\left(z-z^{\prime}\right)$,

with boundary conditions,

$$
\begin{aligned}
& -j \omega \mu_{0} \frac{\left.G_{n}\left(z, z^{\prime}\right)\right|_{z=0}}{\partial G_{n}\left(z, z^{\prime}\right) /\left.\partial z\right|_{z=0}}=Z_{n}^{(1)}, \\
& -j \omega \mu_{0} \frac{\left.G_{n}\left(z, z^{\prime}\right)\right|_{z=t_{s}}}{\partial G_{n}\left(z, z^{\prime}\right) /\left.\partial z\right|_{z=r_{s}}}=Z_{n}^{(3)} .
\end{aligned}
$$

If a Green function is found, $V_{n}(z)$ will be obtained from the integral equation (15). We take advantage of the concepts of circuit theory [9] to find the Green function. Then, the Green function can be written as:

$$
\begin{aligned}
G_{n}\left(z, z^{\prime}\right)=V_{n}^{\prime}(z)= & V^{+} \exp \left(-j k_{z, n}^{(2)}\left(z-z^{\prime}\right)\right) \\
& +V^{-} \exp \left(j k_{z, n}^{(2)}\left(z-z^{\prime}\right)\right),
\end{aligned}
$$

where $V^{+}$and $V^{-}$are the unknown coefficients to be determined, and the corresponding current wave in the transmission line is written in the form

$I_{n}^{\prime}(z)=Y_{n}^{(2)} V_{n}^{\prime}(z)$.

For a point current source, which is excited at $z=z^{\prime}$, the following equation is satisfied,

$V_{n}^{\prime}\left(z^{\prime}\right)=\frac{1}{Y_{n}^{u p}\left(z^{\prime}\right)+Y_{n}^{d n}\left(z^{\prime}\right)}$,

where $Y_{n}^{u p}\left(z^{\prime}\right)$ and $Y_{n}^{d n}\left(z^{\prime}\right)$ are the admittances looking upward and downward from the interface be- tween air and the grating region. In addition, the boundary conditions at $z=0$ and $z=t_{g}$ are written as:

$\frac{\left.V_{n}^{\prime}(z)\right|_{z=t_{8}}}{\left.I_{n}^{\prime}(z)\right|_{z=t_{8}}}=Z_{n}^{(3)}$,

$\frac{\left.V_{n}^{\prime}(z)\right|_{z=0}}{\left.I_{n}^{\prime}(z)\right|_{z=0}}=Z_{n}^{(1)}$.

From (A4) and (A5a), the Green function (as $z \geq z^{\prime}$ ) would be derived in the following form:

$$
\begin{aligned}
& G_{n}\left(z, z^{\prime}\right) \\
& =\frac{\exp \left(-j k_{z, !}^{(2)}\left(z-z^{\prime}\right)\right)+\Gamma_{23} \exp \left(j k_{z, .}^{(2)}\left(z+z^{\prime}-2 t_{g}\right)\right)}{\left(1+\Gamma_{23} \exp \left(-2 j k_{z, .,}^{(2)}\left(t_{g}-z^{\prime}\right)\right)\right)\left(Y_{n}^{\prime \prime}\left(z^{\prime}\right)+Y_{n}^{d \prime}\left(z^{\prime}\right)\right)},
\end{aligned}
$$

where $\Gamma_{23}$ defines the reflection coefficient between grating and substrate, and is represented by $\left(Z_{n}^{(3)}\right.$ $\left.Z_{n}^{(2)}\right) /\left(Z_{n}^{(3)}+Z_{n}^{(2)}\right)$.

\section{References}

[1] K. Handa, S.T. Peng and T. Tamir, Appl. Phys. 5 (1975) 325.

[2] Shuzhang Zhang and T. Tamir, IEEE J. Quantum Electron. QE-29 (1993) 2813.

[3] J.T. Sheridan and L. Solymar, J. Opt. Soc. Am. A 9 (1992) 1586.

[4] T.K. Gaylord and M.G. Moharam, Proc. Inst. Electr. Eng. 73 (1985) 894.

[5] F.G. Kaspar, J. Opt. Soc. Am. 63 (1973) 37

[6] C.B. Burckhardt, J. Opt. Soc. Am. 56 (1966) 1502.

[7] S.T. Peng, H.L. Bertoni and T. Tamir, Optics Comm. 10 (1974) 91.

[8] T. Tamir, Optik 38 (1973) 269.

[9] L.B. Felsen and N. Marcuvitz, Radiation and Scattering of Waves (Prentice-Hall, Englewood Cliffs, N.J., 1973) p. 185. 